Bulletin de

L'A.P.A.D

\section{Bulletin de l'APAD}

15 | 1998

Les dimensions sociales et économiques du développement local et la décentralisation en Afrique au Sud du Sahara

\title{
Les Dimensions Socio-Économiques du Développement Local en Afrique au Sud du Sahara : Quelles Stratégies pour quels Acteurs?
}

Pierre-Joseph Laurent et Jean-Philippe Peemans

\section{OpenEdition \\ Journals}

Édition électronique

URL : http://journals.openedition.org/apad/553

DOI : 10.4000/apad.553

ISSN : 1950-6929

Éditeur

LIT Verlag

Édition imprimée

Date de publication : 1 mai 1998

Référence électronique

Pierre-Joseph Laurent et Jean-Philippe Peemans, «Les Dimensions Socio-Économiques du

Développement Local en Afrique au Sud du Sahara : Quelles Stratégies pour quels Acteurs ? », Bulletin de l'APAD [En ligne], 15 | 1998, mis en ligne le 20 décembre 2006, consulté le 07 septembre 2020. URL http://journals.openedition.org/apad/553; DOI : https://doi.org/10.4000/apad.553

Ce document a été généré automatiquement le 7 septembre 2020

Bulletin de l'APAD 


\title{
Les Dimensions Socio-Économiques du Développement Local en Afrique au Sud du Sahara: Quelles Stratégies pour quels Acteurs?
}

\author{
Pierre-Joseph Laurent et Jean-Philippe Peemans
}

1 Le propos de cette contribution est d'insister sur les dimensions socio-économiques de la décentralisation et du développement local. Elle cherche à établir le lien entre la question d'une construction institutionnelle du territoire et la problématique du local comme lieu de manifestation de conflits entre différents types d'acteurs du développement.

2 La construction institutionnelle d'un territoire affecte la nature du pouvoir, les relations entre ce dernier et l'évolution sociale, les interactions entre celle-ci et les changements économiques. Elle oblige, dans la réflexion sur la décentralisation, à sortir du cadre étroit d'une réforme administrative cherchant à trouver une répartition optimale des compétences entre instances centrales, régionales et locales de l'appareil d'Etat. Elle réinscrit la décentralisation dans le cadre d'une stratégie d'acteurs qui elle-même renvoie aux changements qui impulsent une restructuration des rapports de force internationaux.

3 Une grille d'analyse ouverte de ce genre peut aider à relire en terme de dynamique contradictoire des concepts souvent appauvris par une utilisation décontextualisée, comme par exemple ceux de "secteur informel" ou de "société civile" .Elle devrait permettre de mieux saisir les aspects contradictoires qui traversent des logiques complexes de structuration et de différenciation sociales. Elle devrait contribuer à éclairer le rapport entre pratiques socio-économiques de certaines catégories d'acteurs, formation de classes et formation de "réseaux". Enfin, elle pourrait aussi clarifier certains enjeux liés à la distinction à faire entre accumulation et développement, en évitant une lecture réductionniste des initiatives multiples des populations au niveau des réalités locales du développement. 
Les acteurs de la globalisation et leur vision de la décentralisation et du développement local

4 Il est important de replacer les conflits entre acteurs autour du local dans la dynamique générale de la tendance à la globalisation qui est dominante, dans l'histoire récente, au point de vue économique et surtout financier.

5 Mais la globalisation elle-même doit être aussi analysée comme un rapport de force entre acteurs et pas seulement comme un phénomène économique. Elle n'est pas indépendante de l'évolution du contexte historique et géo-politique des années 1980.

6 Il est très important de savoir établir une liaison entre l'économie politique de l'ajustement et celle de la globalisation. Si on ne le fait pas, on risque de rester enfermé dans une approche fixiste des "pays du Tiers-Monde écrasés par la dette", alors qu'une nouvelle dynamique de différenciation sociale liée à la globalisation se met en place.

7 Si les critères de conditionnalité ont souvent été dénoncés comme imposant des contraintes exorbitantes (démantèlement des services publics, réduction des dépenses de santé et d'éducation et des subsides aux produits de base), on ne peut négliger qu'en même temps ils ont favorisé l'élargissement du rôle de l'entreprise privée, de son autonomie face à l'Etat, et l'ont libérée de nombreuses entraves fiscales et réglementaires.

8 Les années 1990 voient se déployer l'ensemble des effets des évolutions qui ont marqué les années 1980.

9 Certaines catégories d'acteurs ont pu s'émanciper des cadres de l'Etat-Nation, au Sud comme au Nord, et mettre en place des stratégies économiques qui ont un cadre de référence mondial. Des groupes économiques se sont consolidés dans un climat d'agressivité et de compétition plus sévères (Aglietta et al. 1990). Ils se sont internationalisés dans leurs stratégies et leurs structures d'activités (Eden \& Potter 1993).

10 Le système de production dominé aujourd'hui par la globalisation des activités des grandes firmes multinationales, repose sur une grande flexibilité dans l'organisation de la production. En terme d'espace cette flexibilité se traduit notamment par une grande capacité de jouer sur divers centres de production, localisés dans diverses parties du monde, et capables de répondre de manière souple aux besoins variables des centres de coordination de l'entreprise mondialisée. Cela se traduit par la délocalisation de nombreuses activités de production jadis liées rigidement à un site de production centralisé. La décentralisation de l'organisation économique succède donc à la centralisation de cette dernière qui était caractéristique des grandes chaînes de production de type fordiste.

11 La décentralisation économique suppose l'existence de nombreux espaces régionaux ou locaux capables d'accueillir les activités délocalisables (Veltz 1996).

12 Ce point doit être souligné quand on parle du local et de la décentralisation. Au niveau des structures économiques, il existe en effet objectivement, dans le cadre de la tendance à la globalisation, en même temps une évolution vers la déconcentration et la délocalisation des activités jadis centralisées, de production. Le "local" peut donc jouer un nouveau rôle fonctionnel par rapport au redéploiement des activités de production globalisées.

13 On peut donc faire au moins l'hypothèse d'un lien entre d'une part le discours dominant sur la décentralisation et les vertus du local, et d'autre part les pratiques de 
déconstruction institutionnelle de l'Etat-Nation jadis fonctionnel par rapport aux exigences de l'accumulation fordiste (Scott \& Storper).

Cette hypothèse renvoie au problème des fondements socio-économiques de l'idéologie de la globalisation et de son rôle dans les conflits d'acteurs autour de la perception de la place du local dans le développement.

Les stratégies de globalisation cherchent à produire un discours qui légitime l'évolution en cours et qui doit prendre le relai du discours qui permettait la construction d'un consensus social autour de stratégies de croissance économique dans le cadre de l'Etat-Nation.

16 Ce discours présente une très forte normativité et a un caractère particulièrement volontariste et ambitieux. Il associe étroitement la mondialisation technologique à une mondialisation de la "démocratie et du marché". Le marché est présenté comme un vecteur de l'ordre global.

17 La réflexion sur la réforme de l'Etat dans les pays du Sud, la démocratisation, la "gouvernance" et la décentralisation s'inscrit ainsi dans le contexte d'une remise en cause du rôle central de l'Etat dans le développement.

La vision de la " bonne gouvernance" imposée à l'Afrique par les acteurs globaux

18 Cette réflexion sur le rôle de l'Etat a d'abord été suscité par le renforcement des conditionnalités économiques de l'aide au développement, à travers la mise en œuvre des politiques d'ajustement dans les années 1980. A la fin des années 1980, elle a évolué vers un renforcement des conditionnalités politiques.

19 Les thèmes de la réforme de l'Etat, de la décentralisation, de la consolidation de la société civile, de la démocratie et de la promotion du marché sont donc des thèmes liés, notamment mais pas exclusivement à travers le concept de gouvernance (Hyden \& Bratton 1992).

20 Décentralisation et démocratisation s'ajoutent à la fin des années 1980 à l'ajustement pour redéfinir un Etat fonctionnel par rapport au marché.

21 La question de la décentralisation n'est donc pas dissociable d'un certain contexte d'ensemble qui cherche à imposer une certaine conception de la structure du "bon Etat" à des Etats en crise, et de plus en plus dépendants financièrement de l'assistance ou de la bonne volonté des bailleurs de fonds. C'est dans ce contexte qu'apparaissent les concepts de "gouvernance locale" (World bank 1992).

22 La décentralisation est vue comme un moyen d'assurer une gestion plus efficace des services publics, capable de réduire les coûts tout en s'adaptant mieux aux demandes des utilisateurs. La décentralisation est vue également comme un moyen de réduire le déficit des finances publiques, en transférant à la fois certaines recettes et certaines dépenses vers les collectivités locales. Cette dévolution est supposée permettre une réduction des gaspillages liés aux coûts d'une centralisation administrative excessive. Elle est censée également permettre un meilleur équilibre entre recettes et dépenses, grâce à une meilleure visibilité de l'utilisation des ressources, à une plus grande exigence de responsabilité (accountability) et à une moins grande capacité de résistance à la pression fiscale au niveau local.

23 Ces idées s'inscrivent dans le sillage de l'importance qui avait été donnée dans les années 1980 à la participation des utilisateurs de services et de biens collectifs à l'amortissement et au coût de fonctionnement de ces derniers. Une grande partie de la littérature sur le financement des projets était alors centrée sur le problème du 
recouvrement d'une partie ou de la totalité des coûts des projets. A ce niveau micro de la gestion des projets on trouvait donc déjà une référence assez forte à la liaison nécessaire entre production de biens publics et mécanismes de marchés, à travers un système approprié de prix ou de redevance (Peemans 1986).

La participation des populations au développement local avait donc dans cette perspective une connotation assez nettement économique et gestionnaire. Participation et marché (ou quasi-marché) étaient complémentaires voire synonymes. Il s'agissait plutôt de pédagogie de participation au marché que de participation active à la vie politique ou à la décision en matière de choix collectifs.

L'affirmation plus ambitieuse d'une volonté de réforme de l'Etat à la fin des années 1980, a élargi le champ d'application de ces idées, tout en restant dans la même perspective économique. Les critères de gestion économique et de marché ne sont plus seulement appliqués au niveau micro des projets, mais il sont étendus au niveau macro, à la réflexion sur la gestion de l'Etat lui-même.

Une partie importante des idées sur la décentralisation vont être inspirées par cette approche. Le local est vu comme pouvant étendre les critères de gestion des projets à la gestion d'un espace politico-administratif plus vaste, et comme pouvant incorporer la logique de marché aux rapports entre producteurs et utilisateurs de services.

27 Mais le succès de l'idée de décentralisation vient aussi sans doute de ce que ce concept est suffisamment large et flou pour pouvoir incorporer à un moment donné des perceptions diverses de nouvelles réalités. Elles donnent l'impression à diverses catégories d'observateurs et d'analystes qu'un ensemble convergent d'éléments disparates incitent à aller vers une nécessaire revitalisation de la dimension locale du développement.

Parmi ces perceptions on peut citer l'importance de plus en plus grande donnée aux réalités du secteur informel, aux initiatives de certaines catégories sociales pour affronter les conséquences de l'ajustement et les tendances croissantes à la paupérisation et à la marginalisation, et également tous les discours sur la démocratisation (Peemans el al. 1996a).

Dans ce contexte le concept de décentralisation qui était initialement réduit un peu étroitement à des considérations de gestion administrative ou budgétaire va pouvoir être enrichi de connotations sociologiques et de références politiques.

A travers cette évolution il y a certainement une ouverture du concept de décentralisation vers de nouvelles dimensions qui finalement en font un enjeu non seulement de la réforme de l'Etat, mais aussi du style de développement. Cette ouverture du concept a rendu donc son utilisation plus pertinente, mais aussi plus complexe, et surtout plus ambiguë.

31 Cependant, pour certains observateurs, la démocratisation imposée après l'ajustement est fondée sur un scenario fort problématique. puisque l'on propose de démocratiser un Etat" ajusté" qui a pratiquement perdu la possibilité de définir ses choix de développement, théoriquement l'objet même des enjeux de la démocratie.

M. Klein a souligné le caractère paradoxal des rapports entre ajustement, démocratie et rôle des nouvelles élites africaines. Le paradoxe selon Klein vient de ce que l'ajustement structurel, dans son essence est une prise de contrôle des économies des pays africains par des institutions étrangères. Ce contrôle extérieur des économies africaines a 
généralement entraîné un programme de désindustrialisation, le licenciement massif de fonctionnaires, la réduction des dépenses en matière de santé et d'éducation.

Mais ces programmes sont défendus par une nouvelle élite africaine, revenue de l'extérieur prendre le contrôle des processus démocratiques. et pour qui la démocratie doit servir à légitimer l'ajustement... Le résultat, dit Klein, est que en Afrique, comme en Europe de l'Est, la démocratisation est identifiée à la forme la plus prétentieuse de l'idéologie du marché (Klein 1992).

Les propos de Klein permettent de relativiser l'approche normative de la réforme de l'Etat africain et de ses rapports avec l'émergence éventuelle d'un noyau d'accumulation tel que souhaité par les "global players".

On ne peut pas se contenter de faire une lecture purement normative de ces contraintes et impulsions ou injonctions internationales. Opposer différentes versions, contradictoires, du "bon" Etat, instrument de "l'accumulation vertueuse" contre la "déviance africaine" mène à une impasse théorique et pratique.

Les résultats visibles de l'ajustement et de la démocratisation obligent en effet à tempérer considérablement, dans les faits, les promesses des grands discours normatifs sur la transition au marché et à la démocratie. D'autres scénarios que le scénario normatif abstrait paraissent pouvoir mieux rendre compte des réalités de l'"économie politique" de l'ajustement et de la démocratisation, dans le cadre de la globalisation.

L'importance "d'une économie politique" de l'ajustement, de la démocratisation et du développement local en Afrique

Une "économie politique" de l'ajustement et de la démocratisation en Afrique doit à la fois tenir compte des changements affectant les rapports internationaux des élites africaines dans le cadre des nouvelles structures de "l'économie politique" de l'accumulation globalisée, et de l'incidence de ces changements sur les relations entre les composantes anciennes et nouvelles de ces élites.

L'évolution des années 1980 a permis d'établir de nouvelles relations entre les réseaux de pouvoirs informels et les appareils d'Etat affaiblis. L'articulation entre les nouvelles formes et les anciennes formes de pouvoir est au cœur des jeux politiques locaux. Le déplacement partiel des enjeux économiques et politiques vers les bases locales de l'accumulation et du développement ne doit pas être abordé à travers une approche idyllique du "local" comme lieu évident de la reconstitution du lien social, de la participation politique, ou encore comme place privilégiée des initiatives de la "société civile". Au niveau de l'identification des besoins, le problème est de savoir si la "demande locale" exprime vraiment les attentes des différents groupes de la population locale. Le plus souvent les priorités sont établies par ceux qui dominent les instances de délibération locales. Les opportunités de s'exprimer "à la base" n'empêchent pas l'existence d'inégalités importantes entre les différentes catégories sociales.

39 Les réalités sont souvent bien loin du "monde enchanté de la solidarité". Les réseaux de sociabilité, basés sur des liens de parenté, de voisinage sont le support de nombreux systèmes d'échanges, de dons et contre-dons, de transferts au sein des collectifs familiaux et lignagers. Ces transferts ne se fondent pas sur une logique égalitaire, car ils ont pour but le plus souvent d'assurer la reproduction de liens sociaux de dépendance et des positions sociales antérieures. En fait, ils doivent être analysés dans le cadre des 
rapports de force, toujours mouvants et entrecroisés, entre "réseaux clientèlistes verticaux" et "réseaux associatifs horizontaux de l'économie populaire".

Prendre en considération les ambiguïtés et les contradictions des rapports entre accumulation et développement permet d'éviter une lecture naïve des initiatives locales des populations rurales et urbaines, lieu privilégié pour certains de la constitution d'une "société civile" exemplaire.

Cela permet aussi de mieux saisir les enjeux portés et perçus par les différents acteurs dans le contexte particulier des années 1980-1990.

Ce que les organisations locales ont en commun c'est d'être souvent l'oeuvre d'une certaine élite intellectuelle, essayant le plus souvent de se positionner d'une manière nouvelle dans le jeu politique, à la faveur du rétrécissement de l'emprise de l'Etat (Goros 1994).

Les dimensions complexes du changement apparaissent mieux lorsqu'on l'explore à partir d'une grille de questionnement ouverte qui met en perspective les enjeux en termes d'accumulation et de développement, et les stratégies des acteurs qui les portent. Le déplacement de ces enjeux du niveau national vers le niveau local est porteur de nouveaux conflits entre initiatives des entrepreneurs "politico-économiques" des nouvelles formes d'accumulation, et populations locales dont les demandes de développement ne coïncident pas nécessairement avec la réponse que ces initiatives constituent. Les conflits autour des conditions d'accès à la maitrise des ressources locales (terre, eau, forêts) s'en trouvent, par exemple, souvent accentués.

44 Mais cette diffusion même des enjeux conflictuels de l'accumulation et du développement contribue à leur donner une plus grande visibilité dans la société, et à ne pas les enfermer dans l'opacité des stratégies de pouvoir intra-élite, masquées derrière des choix imposés du haut, comme des exigences de la logique d'Etat.

Cela permet aussi de replacer les enjeux du local dans une perspective globale.

Les logiques de "réseaux" et de "territoire" recoupent une recomposition des rapports entre groupes sociaux ,une restructuration de "rapports de classe" aux contours encore indécis, mais qui est bien sous-jacente et que certains contextes peuvent soit exacerber, soit euphémiser. Derrière cette recomposition se profile une redéfinition du rôle de l'Etat dont l'enjeu est de savoir finalement de quel groupe ou de quelle coalition d'intérêt il sera l'instrument de pouvoir à l'avenir.

47 La dynamique de recomposition des rapports de force au niveau global vient interférer avec les évolutions locales, la crise des Etats-Nations affaiblissant leurs capacités à moduler ces interactions directes entre le global et le local. Ce sont désormais celles-ci qui, bien souvent, ont gagné un espace accru pour redessiner ensemble le rôle et les fonctions assignées à l'Etat.

Ce sont ces composantes socio-économiques et socio-politiques qui permettent d'éclairer les conflits entre acteurs, sous-jacents aux discours et aux pratiques concernant la décentralisation. Derrière l'unanimité sur l'intérêt et la nécessité de la décentralisation, il y a de profondes divergences sur le contenu et le sens de la décentralisation.

Il y au moins trois catégories d'acteurs qu'on a eu le tort de regrouper un peu facilement dans le "panier" de la "société civile", parce que dans les années 1980, ils avaient en commun une méfiance ou une opposition par rapport aux pratiques des 
régimes autoritaires de toutes tendances. Ils ont des positions divergentes à propos de la décentralisation.

Il y a d'une part des groupes que l'on peut identifier à une élite urbaine se retrouvant surtout dans les milieux professionnels et académiques, très ouverte vers l'international, voire liée par la consultance ou la carrière à des organisations internationales. A travers des circonstances diverses selon les pays, mais convergentes, une partie de cette élite avait été écartée des affaires ou marginalisée dans certains cas par les régimes en place, à mesure que ceux-ci voyaient leur support se réduire à des cercles étroitement liés aux circuits de redistribution clientéliste dépendant du pouvoir. Ces élites avaient donc atteint un certain degré d'autonomie, par rapport aux régimes en place, et ne se gênent plus pour exprimer leur insatisfaction ou leur mépris quant au style prédominant de gestion des affaires publiques.

Il y a d'autre part les groupes que l'on peut identifier à une partie du monde des cadres intermédiaires de la fonction publique, et des intellectuels qui ont perdu leur emploi ou été laissés sans avenir professionnel, suite à la contraction du secteur public dans les années 1980. Ils ont progressivement rejoint les cadres des Eglises ou d'ONG nationales, impliqués dans des projets soutenus et financés par des ressources extérieures depuis la fin des années 1970. Le développement de nouvelles associations, de nouvelles ONG, ou l'élargissement d'ONG existantes étaient une des seules voies ouvertes pour développer l'auto-emploi de ces cadres intermédiaires, souvent d'origine urbaine, mais prêts à se reconvertir dans des projets de développement en monde rural, pour autant que cela permette d'obtenir des financements extérieurs. Cette catégorie d'individus est aujourd'hui en expansion à la faveur de l'invention de la coopération décentralisée, qui travaille sans plus passer par des représentants de l'Etat.

Enfin il a tous les groupes (les plus nombreux), s'organisant à partir des pratiques paysannes et des classes populaires urbaines, qui ont pour objectif essentiel la défense et l'amélioration des conditions de vie dans un milieu concret. Cette dernière catégorie d'acteurs n' a pas nécessairement la même vision de ce que doit être le "pouvoir local" que le second groupe d'acteurs mentionné ci-dessus, et à fortiori que le premier groupe.

Pratiques et demandes populaires de développement local

Les groupes qui s'organisent à partir des pratiques paysannes et des classes populaires urbaines, ont pour objectif essentiel la défense et l'amélioration des conditions de vie dans un milieu concret. Cette dernière catégorie d'acteurs a d'autres attentes vis-à-vis du "pouvoir local" que les diverses catégories d'élites qui veulent augmenter leur capital social et économique à travers la décentralisation.

De nombreuses analyses, centrées sur les pratiques socio-économiques concrètes des populations ont reconnu progressivement que les populations locales se montrent capables, dans certains cas, d'inventer un ensemble de règles et de les rendre effectives, dans le cadre d'un espace clairement identifié. L'invention du local consiste, avant tout, dans la maîtrise d'un nouvel espace. Celle-ci comprend aussi bien les réalités du secteur informel urbain, les pratiques associatives, les formations de groupements pré-coopératifs en milieu rural, les groupements de femmes et de jeunes, que la multiplication des réseaux d'affaires mêlant les pratiques ethno-clientélistes avec des initiatives économiques à dimensions locales, régionales, nationales et internationales. Il faut donc reconnaître la vitalité extraordinaire des sociétés africaines, dans les pratiques des milieux urbains et des populations rurales (Laurent \& Peemans 1998). 
La reconstruction du développement local par la petite paysannerie simples critères d'entrée sur le marché. C'est là une grande différence avec les approches néo-libérales qui évaluent le monde paysan à partir de critères de fonctionnalité économique par rapport aux exigences de l'accumulation aujourd'hui globalisée. C'est là aussi que se mesure toute la différence entre la demande des acteurs du "petit marché populaire" et l'offre d'ajustement des agents de l'accumulation normalisée à l'échelle mondiale. Le monde paysan doit être approché aussi en terme d'acteurs dont les stratégies sont mues à la fois par des valeurs culturelles et par une volonté de s'adapter à des contraintes externes de plus en plus pesantes et envahissantes. exemple les initiatives des femmes dans le petit commerce ou la production agricole apparaissent comme ayant un caractère individualiste en opposition ou même en rupture avec l'ordre lignager ancien et ses pratiques agricoles communautaires combinées avec des systèmes de redistribution du produit sous l'égide des anciens. Cette évolution semble confirmée par celle de l'attitude à l'égard de l'épargne, ou l'on voit les femmes rurales pauvres chercher à protéger le revenu tiré de leurs activités agricoles ou commerciales des formes anciennes de redistribution à l'intérieur du lignage, en déposant ce revenu dans l'une ou l'autre sorte de caisse d'épargne locale.

Ces tendances conduisent certains observateurs à voir dans ces pratiques une demande d'émancipation du poids des traditions communautaires qui conforterait les choix de politique faits en faveur d'une insertion individuelle des franges les plus pauvres de la population dans le marché. Dans cette optique, les causes fondamentales de la pauvreté résident à la fois dans le manque d'opportunité pour s'insérer dans le marché et dans les blocages multiples inhérents à la société traditionnelle. Parmi ceux-ci figurent notamment les contraintes de redistribution qui pèsent sur ses membres, empêchant la formation de l'épargne et la prise d'initiatives économiques permettant d'élever le revenu. Les politiques de lutte contre la pauvreté devraient dès lors, dans cette optique, être axée sur la capacité de s'insérer dans le marché à travers la mise en œuvre de systèmes de micro-crédit ciblés et appropriés, permettant de stimuler l'apparition de micro-entreprises et de micro-entrepreneurs, femmes et hommes.

Cependant, les stratégies paysannes sont complexes et visent avant tout la reproduction de la famille paysanne. Les aspects économiques sont importants et les initiatives sont réelles. Mais il serait cependant tout à fait inadéquat de limiter les comportements paysans à des stratégies plus ou moins réussies d'insertion sur le marché. Si les femmes revendiquent un rôle croissant dans l'économie marchande, on voit également qu'elles ont un poids essentiel dans le maintien des conditions de reproduction des familles.

C'est à travers l'observation de la place des femmes dans la société que l'on peut voir comment une insertion dynamique dans le "petit marché" est totalement encastrée dans les stratégies paysannes de reproduction de la famille. Les femmes paysannes 
jouent donc un rôle prépondérant dans la mise en œuvre d'un "modèle paysan de développement" .

La nécessité d'une prise en considération les pratiques associatives paysannes dans la construction institutionnelle de la décentralisation

61 Les perspectives esquissées ci-dessus permettent de situer l'importance des enjeux socio-économiques de la décentralisation dans les régions rurales et de ce qui a été appelé par certains la "gouvernance locale". Le "modèle de développement paysan" est non seulement capable d'évolution et d'intensification au sens boserupien, il est aussi au cœur d'un mode de développement "durable et humain".

Il s'agit de mettre en place un ensemble d'institutions au niveau local qui puissent établir un ensemble de synergies entre les initiatives des associations paysannes et les cadres d'une coopération entre les communautés, les villages pour réaliser un ensemble de travaux qui puissent créer les bases d'une amélioration du cadre de vie. Il s'agit de mettre en place, en même temps, un cadre institutionnel qui puisse renforcer le pouvoir de négociation des collectivités organisées face à l'Etat et aux bailleurs de fonds.

63 Cette évolution aide à la fois à redéfinir les conditions du développement, en tenant compte des demandes et des pratiques des populations, et à envisager la mise en place d'institutions qui peuvent aider à consolider ces initiatives.

64 Ces institutions doivent donc avoir aussi une dimension socio-économique. Ce sont en effet les initiatives socio-économiques des populations qui leur permettent de construire leur identité à travers l'amélioration de leur cadre de vie. Ces initiatives peuvent aussi faciliter la mise en place de liens de coopération entre divers groupes de population dans une même région. Cette évolution peut donc aider à réduire les tensions liées à l'exacerbation d'une définition strictement culturelle de l'identité (comme certaines formes de réinvention de l'ethnicité ; cf. Peemans et al. 1996b) et contribuer à la fois à la stabilité politique et au développement des populations en tenant compte de leurs demandes de développement.

La décentralisation doit donc prendre en considération la nature des initiatives et des demandes de développement économiques et social des populations. Elle doit mettre en place des institutions qui permettent de redéfinir un "nouveau pacte" entre ces populations et l'Etat, comme base de reconstruction du politique dans le respect de la trajectoire historique des différentes régions.

66 La reconnaissance de l'identité et des demandes du monde paysan supposent la prise en considération de stratégies qui peuvent conforter les initiatives paysannes.

67 En fait les pratiques paysannes montrent qu'un tout autre sens peut être donné à la décentralisation si l'on veut qu'elle tienne compte des demandes de développement des populations, révélées non pas par des discours mais par des pratiques. On voit émerger lentement de nouvelles demandes institutionnelles dont les pratiques populaires sont le révélateur et qui combinent une logique d'association et des initiatives locales visant à améliorer les conditions de vie.

Selon les observateurs qui connaissent ces réalités, mieux cibler un soutien à la petite paysannerie, requiert que ce soutien soit centré sur les structures spécifiques des communautés paysannes locales et sur la nature et les problèmes des éco-systèmes qui les entourent. 
69 L'impression dominante des observations faites dans le cas du Burkina Faso tend à montrer que l'insertion recherchée dans le marché s'accompagne en même temps d'un foisonnement d'initiatives qui vont dans le sens de la réinvention d'un lien social aux dimensions multiples, combinant à la fois la solidarité, l'individualisme, le clientélisme, la concurrence et de nouvelles formes associatives. L'aspect intégré des diverses composantes des initiatives locales et la demande de sécurisation des populations sont des réalités qui se renforcent les unes les autres, et qui paraissent être des dimensions essentielles des pratiques d'économie populaire.

70 Reconnaître l'expérience, les besoins, attentes et potentialités des structures sociales anciennes et nouvelles (assemblées de village, associations paysannes, associations de femmes, groupements de jeunes) est un facteur important pour substituer une relation quasi-contractuelle à une relation autoritaire et améliorer les chances de participation.

71 La mise en place d'un cadre de décentralisation appropriée doit veiller à conforter l'élaboration d'un cadre institutionnel qui renforce les synergies entre les différents types d'organisations informelles existant actuellement. On peut aider ces groupes informels à se donner une organisation adéquate pour participer à l'exécution et à la gestion, en en faisant le support de structures décentralisées et intégrées au niveau local pour l'octroi de crédit, la mobilisation de l'épargne, l'achat d'équipement et d'intrants, et la commercialisation des produits.

72 Mais ce cadre institutionnel ne pourra être adapté que s'il résulte dans chaque cas d'un processus lent, respectant l'esprit et les contraintes du contexte local, et se situe dans le prolongement de la trajectoire historique du développement local. La construction institutionnelle du "territoire" ne peut résulter que d'une maturation lente, exprimant l'identité locale ; elle ne peut donc être imposée du haut comme une sorte d'application locale d'un modèle général.

73 Dans ces montages institutionnels, une organisation coopérative, prolongeant les initiatives actuelles du monde paysan peut jouer un rôle important, à condition de bien mesurer les difficultés et les ambigüités révélées par l'histoire, déjà longue, des expériences coopératives en Afrique.

Pistes pour un appui au local dans la cadre de la décentralisation de l'Etat et de la coopération

74 L'appui à l'économie populaire instituée ne pourra faire l'économie d'une réflexion sur les modalités de soutien à ce type d'associations non spécialisées, - qualifiées trop hâtivement par certains de fourre tout, de bricolage, dilettantisme -. Elles abritent et élaborent une partie de l'économie instituée et les actions qu'elles proposent n'ont finalement que peu de chose à voir avec les formules d'appui à l'organisation et au financement du monde rural.

75 Une tentative d'élaboration d'un appui aux associations populaires dans le cadre de la décentralisation de l'Etat et de la coopération, devrait articuler un rapport entre deux composantes de la société. Renforcer l'une, c'est-à-dire l'Etat ou les groupes locaux, à l'exclusion de l'autre conduirait à l'exacerbation des tensions. Cette pratique risquerait d'activer des oppositions pouvant conduire à une remise en cause d'équilibres précaires. On insistera sur le fait qu'une politique d'appui qui, dans un contexte de forte dualisation de la société, viserait essentiellement à rendre l'Etat fonctionnel par rapport au marché et non pas de tenter de rapprocher ce même Etat des pratiques qu'il a toujours considéré comme informelles - de gestion de l'espace local 
concrètement mise en œuvre par les associations populaires, produirait en même temps des facteurs d'instabilité. Voulant les combattre, les politiques qui ne tiendraient pas compte des stratégies des groupes d'acteurs, qui composent la société, deviendraient la cause de cette instabilité.

Dans cette perspective, la recherche de modalités d'appui à l'économie populaire instituée, telle que partiellement mise en œuvre par des associations populaires, devient une opportunité de relégitimer l'Etat, à travers une nouvelle lecture de l'articulation entre le global et le local. A la faveur d'une proposition institutionnelle raisonnée qui attribue à chaque acteur de l'arène locale une position particulière dans le développement, il est indirectement question de repenser des modalités d'intervention d'un État, non plus basées sur des principes largement importés, mais sur sa légitimation par les populations, à la faveur de la reconnaissance de l'existence d'affaires locales, c'est-à-dire concrètement en procédant à la "formalisation" des pratiques de sécurisation mises concrètement en œuvre par des groupes de populations.

Dans le cadre de la coopération décentralisée, il est question de concevoir un montage institutionnel qui renforce, par le biais d'un financement externe, la négociation entre les parties. Cette proposition s'oppose à la voie classique de soutien formulée dans le schéma suivant: bailleurs de fonds $\Rightarrow>$ partenaires étatiques $\Rightarrow>$ bénéficiaires, aujourd'hui remplacée - suite aux analyses qui jettent sans nuance l'opprobre sur l'Etat -par la proposition: bailleur de fonds => bureaux d'études ou structures privées $\Rightarrow$ bénéficiaires. Cette ultime proposition non seulement écarte l'Etat de la décision, mais considère les associations populaires comme des spectateurs. Notre position, par contre, invite l'Etat et plus concrètement ses représentants provinciaux (et les bailleurs de fonds) à prendre en considération les pratiques de développement des populations à un niveau local et à participer à leur mise en œuvre.

A titre simplement prospectif, une proposition d'appui pourrait s'articuler autour d'un schéma institutionnel, organisé en quatre pôles. 1) Un cadre de concertation: regroupement d'associations, d'unions, de coopératives ou de fédérations paysannes au sein d'un espace local. Cette structure indépendante serait gérée par des représentants des associations adhérentes. 2) Une structure d'appui : institution permanente qui émanerait du cadre de concertation. Elle constitue un organe de conseil et d'appui, au service du cadre de concertation. Le Cadre de Concertation et la Structure d'Appui constituent un tandem inséparable, maîtrisé statutairement par les représentants des associations de la concertation. Ces deux institutions seraient des structures juridiquement reconnues, émanant de la société locale. 3) Un Comité de Pilotage: structure mixte regroupant des représentant a) de l'Etat: ministères, administration, services techniques, b) des Associations locales, c) des donateurs. Le Comité de Pilotage, sur base des dossiers déposés par le Cadre de Concertation statuerait sur le bien fondé d'un financement. Le Comité de Pilotage gérerait le fonds d'appui à des actions liées à l'économie sociale (par exemple, un fonds d'appui régional au développement de l'économie sociale). Le mode de représentation, en vigueur au sein de cette instance, accorde une part égale de sièges aux grands groupes qui composent la société au niveau local. Cette proposition vise à tenir compte des prérogatives de l'Etat, tout en accordant au monde associatif un droit de regard sur les financements qui leur sont destinés et sur la politique de développement à un niveau local. 4) Une Cellule Technique et de Contrôle : structure permanente d'appui au Comité de Pilotage. 
La notion de décentralisation, dont il est aujourd'hui largement question en Afrique, conduit à une redéfinition entre l'Etat - à travers la notion de collectivités locales décentralisées - et ses populations d'une part et entre l'Etat et la coopération au développement d'autre part. S'interroger sur l'articulation entre le global et le local dans le cadre de la souveraineté nationale et du rôle de la coopération décentralisée est donc capital. Il s'agit moins de réduire l'Etat à une portion congrue que de penser sa re-légitimation par les groupes qui composent sa population. Dans cette perspective, la recherche de modalités d'un appui adéquat à l'économie populaire instituée jouera un rôle déterminant, par sa participation à la perpétuation d'un Etat "viable" reposant sur un dialogue avec ses populations, dans un cadre légal institué.

\section{BIBLIOGRAPHIE}

Aglietta, M., A. Brender \& Y. Coudert 1990. Globalisation financière : l'aventure obligée. Paris : Economica.

Eden, L. \& E. Potter (eds.) 1993. Multinationals in the Global Political Economy. New York: St.Martin's Press.

Gorus, J. 1994. In : Administration Générale de la Coopération au Développement : 71-73.

Hyden, G. \& M. Bratton (eds.) 1992. Governance and Politics in Africa. Boulder : Lynne Rienner Publishers.

Klein, M.A. 1992. "Back to Democracy" ; Presidential Address to the 1991 Annual Meeting of the African Studies Association. African Studies Review 35 (3) : 6-8.

Laurent. P.-J. \& J.-P. Peemans 1998. Dynamiques d'économie sociale : pratiques locales et rôles des aides extérieures. Louvain-La-Neuve : Institut d'Études du Développement-CIUF-AGCD.

Peemans, J.-P. 1986. Politiques et procédures financières intéressant le développement rural. Rome : FIDA.

Peemans, J.-P., A. Esteves \& P.-J. Laurent 1996b. Stabilité politique, ethnicité et dimensions socio-économique de la gouvernementalité locale. Bruxelles : AGCD.

Peemans, J.-P., A. Esteves \& P.-J. Laurent, 1996a. Stabilité politique, ethnicité et dimensions socio-économiques de la gouvernementalité locale : synthèse théorique et application, les cas du Bénin et du Burkina Faso. Louvain-La-Neuve : Institut d'Étude du Développement-CIUF -AGCD.

Scott A. \& M. Storper, "Le développement régional reconsidéré". Espaces et sociétés 66-67 : 7-37.

Veltz, P. 1996. Mondialisation, villes et territoires. Paris : Presses Universitaires de France. World Bank 1992. Governance and Development. Washington : World Bank. 


\section{AUTEURS}

\section{PIERRE-JOSEPH LAURENT}

Unité d'Anthropologie de l'Université Catholique de Louvain, Place Montesquieu 3. B-1348 Louvain-La-Neuve (Belgique). Tél. +32 (64) 6774 39. Fax. +32 (10) 472805 laurent@dvlp.ucl.ac.be

JEAN-PHILIPPE PEEMANS

Institut d'Etudes du Développement de l'Université Catholique de Louvain. 